FOCUS

\title{
2011 Showa Ilka Award Technique and results of "meshplating", a method of spondylolysis repair for spondylolysis and spondylolytic spondylolisthesis
}

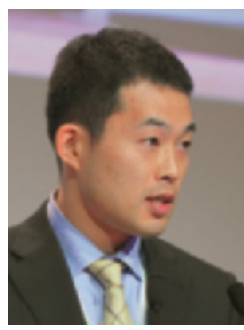

_J. Tonosu, S. Sano, M. Kimura, N. Kawamura, R. Kono, T. Doi, T. Kawai

Orthopedic Department, Sanraku Hospital Chiyoda-Ku, Tokyo, Japan

A technique of "meshplating" was performed on 18 patients with spondylolytic spondylolisthesis and on 9 patients with spondylolysis. The "meshplate" was made of titanium mesh and composed of a cylinder part and a plate part. The "meshplating" was the method of spondylolysis repair, filling the cylinder part with cancellous bone and attaching the plate part to the lamina with mini-screws. It was performed by inside-repair for all cases of spondylolysis and 9 cases of spondylolytic spondylolithesis, and by outside-repair for 13 cases of spondylolytic spondylolithesis. In all cases, the mesh-plated laminae were fixed by the modified Scott method; pedicle screws and wire fixation. The mean follow-up period was 39 months. The results seemed to be excellent with a mean JOA score improvement of $80 \%$. There was no sign of pseudoarthrosis, adjacent segment degeneration, or neurological complication in spite of some implant failures. The outside-repair technique provided less stability of the mesh-plated lamina than inside-repair, because the lamina had been completely separated from surrounding soft tissues. It may be effective to connect the mesh-plated lamina with the caudal spinous process by wiring or spinous process plates when stability is insufficient. • 